\title{
Fusion hindrance in heavy systems with time-dependent Hartree-Fock
}

\author{
Kouhei Washiyama ${ }^{1, \star}$ \\ ${ }^{1}$ Center for Computational Sciences, University of Tsukuba, Tsukuba 305-8577, Japan
}

\begin{abstract}
We analyze fusion hindrance in heavy systems, where the fusion probability around the Coulomb barrier is hindered compared with that in light and medium-mass systems. We perform simulations of central collisions around the Coulomb barrier in heavy systems with time-dependent Hartree-Fock (TDHF) and find that the fusion hindrance is realized in TDHF simulations. We extract nucleus-nucleus potential and energy dissipation in heavy systems by a method combining a microscopic TDHF evolution to a macroscopic collective equation of motion. We find that the extracted potentials exhibit a dynamical increase at small relative distances, while the extracted friction coefficients show rather a behavior similar to that in light and medium-mass systems. We find from our analysis that the dynamical increase in potential is a main contribution to this fusion hindrance.
\end{abstract}

\section{Introduction}

The synthesis of superheavy elements by using heavyion fusion reactions has been a major subject in nuclear physics. The discovery of superheavy elements gives an impact in our society. Especially in Japan, a great impact has been given to a wide area of Japanese society through major newspapers and TV news since Nihonium was discovered and named.

The discovery of such superheavy elements has been realized by a recent progress on experimental technique in heavy-ion fusion reactions. From the theory side, we need to predict suitable combinations of projectile and target, and suitable bombarding energies to help the experimental side to design future experiments. However, lack of understanding the whole process of superheavy element synthesis that is from the entrance channel to survival of evaporation residue against fission. is still significant.

In this proceedings, we focus on fusion process from the capture (touching of projectile and target) and compound nucleus formation. It is known for long as a fusion hindrance ${ }^{1}$ that fusion probability of heavy systems (whose charge products are larger than approximately 1,600) at energies around the Coulomb barrier is significantly hindered compared with that of light and medium-mass systems [1-3]. This hindrance was predicted and extensively discussed by Swiatecki and his collaborators with a model based on the macroscopic liquiddrop model [4-6]. In these seminal works, potential landscape and energy dissipation were key ingredients to understand the mechanism of the fusion hindrance. Later on, Langevin dynamical models have been developed and ap-

\footnotetext{
^e-mail: washiyama@nucl.ph.tsukuba.ac.jp

${ }^{1}$ Note that the fusion hindrance explained in this proceedings is different phenomenon from the fusion hindrance appearing at deep subbarrier energies in light and medium-mass systems, which were discussed by several authors during the conference.
}

plied to the analysis of the synthesis of superheavy elements such as competition of the compound nucleus formation and quasi-fission process [7, 8].

We would like to address the origin of the fusion hindrance from the microscopic point of view. The aim of the present work is therefore to analyze the fusion hindrance phenomena by a microscopic reaction model capable of treating fusion reactions around the Coulomb barrier energy. For this aim, we employ the time-dependent Hartree-Fock method as a microscopic reaction model. Furthermore, to understand the fusion hindrance from the potential landscape and energy dissipation mentioned above, we employ a method that maps the time evolution of a microscopic system that has many internal degrees of freedom to a macroscopic equation of motion that has only a few collective degrees of freedom, which we proposed $[9,10]$. As a result, this method extracts nucleus-nucleus potential and energy dissipation from collective degrees of freedom to internal degrees of freedom. In the following, we will explain our method of extracting potential and energy dissipation and show these results in heavy systems. Then, we will show that the obtained dynamical increase in potential is a main origin of the fusion hindrance.

\section{Method}

The time-dependent Hartree-Fock (TDHF) method is a powerful method to describe nuclear collective motion far from equilibrium. The method can treat both static and dynamical properties of nuclei in a self-consistent framework. Since the first application of TDHF [11] in 1970, many applications of TDHF to nuclear reactions have been performed. Recent progress due to powerful computational facilities allows us to perform TDHF simulations with full Skyrme energy density functionals and without restriction on spacial symmetries. Along this line, we 
briefly explain our method of extracting nucleus-nucleus potential and friction coefficient from TDHF simulations, which consists of the following steps: i) First, we perform TDHF simulations for central collisions to obtain the time evolution of densities of a colliding system. ii) From the obtained densities, we divide the colliding system into projectile-like and target-like fragments by properly defining a neck position. We define a neck position as the position at which one-dimensional density $\rho(x, y=2 \mathrm{fm}, z=$ $0 \mathrm{fm}$ ) along the collision axis ( $x$ axis) becomes minimum [12]. iii) We compute the center-of-mass positions of each fragment, denoted by $R_{1}$ and $R_{2}$, and associated momenta of each fragment, denoted by $P_{1}$ and $P_{2}$. We also compute the masses of each fragment by $m_{i}=P_{i} / \frac{d R_{i}}{d t}(i=1,2)$, and the reduced mass $\mu$ of the system. iv) We construct time evolution of relative distance $R$ and relative momentum $P$ from $R_{1}, R_{2}, P_{1}$ and $P_{2}$. v) We assume that the obtained time evolution of the collective variables $R$ and $P$ obeys the following classical macroscopic equation with a friction term,

$$
\begin{aligned}
& \frac{d R}{d t}=\frac{P}{\mu}, \\
& \frac{d P}{d t}=-\frac{d V}{d R}-\frac{d}{d R}\left(\frac{P^{2}}{2 \mu}\right)-\gamma \frac{P}{\mu},
\end{aligned}
$$

where $V$ and $\gamma$ denote nucleus-nucleus potential and friction coefficient, respectively, which are considered as unknown quantities. These quantities may depend on the relative distance $R$. vi) To obtain two unknown quantities $V(R)$ and $\gamma(R)$, we prepare two trajectories for two slightly different center-of-mass energies under the assumption that $V(R)$ and $\gamma(R)$ do not change by this slight change of energy. Then, to solve the system of two equations at $R, \gamma(R)$ is obtained as

$$
\gamma(R)=-\frac{\dot{P}_{I}-\dot{P}_{I I}}{\dot{R}_{I}-\dot{R}_{I I}}+\frac{\dot{R}_{I}+\dot{R}_{I I}}{2} \frac{d \mu}{d R},
$$

where subscripts $I$ and $I I$ indicate quantities at two slightly different energies $E_{I}$ and $E_{I I}$, respectively. Accordingly, $V(R)$ can be obtained. We take $E_{I I}-E_{I}=0.4 \mathrm{MeV}$ when $E_{I}$ is the fusion threshold energy above which fusion occurs and below which fusion does not occur. In the following, the nucleus-nucleus potentials, friction coefficients, and reduced masses are extracted at the fusion threshold energy, except for $E_{\mathrm{cm}}=250 \mathrm{MeV}$ in Fig. 1 and ${ }^{70} \mathrm{Zn}+{ }^{208} \mathrm{~Pb}$ system in Fig. 3 where we take $E_{I I}-E_{I}=$ $2.0 \mathrm{MeV}$.

We would like to note that the extraction described above is not possible when the following situations are encountered: (i) the denominator in Eq. (3) becomes close to zero, (leading to divergence of the friction coefficient), (ii) the velocity of a fragment becomes close to zero, (leading to divergence of its mass), (iii) the neck position can not be properly determined (leading to difficulty of constructing two-body kinematics). We have encountered these situations in many systems considered when the overlap of projectile and target nuclei becomes significantly large at the later stage of the fusion process. Once we face one of the above situations at a certain relative distance, we stop the extraction. We denote this relative distance as $R_{\min }$.

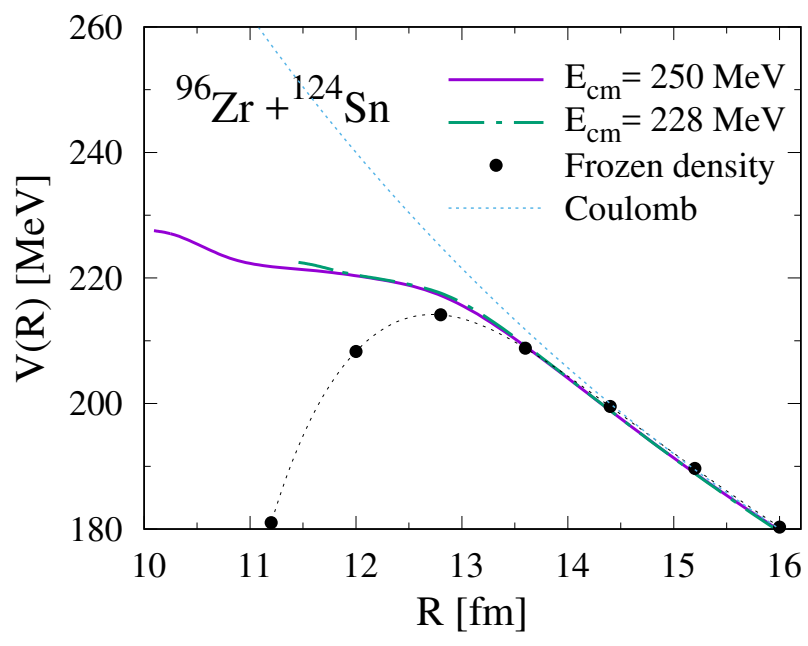

Figure 1. Nucleus-nucleus potentials as a function of $R$. The solid and dot-dashed lines are from our method at two different energies, while the solid circles are the result of the frozen density potential. The dotted line shows the pure Coulomb potential for reference.

We perform the TDHF simulation with Skyrme SLy4d parametrization [13]. Pairing correlations are neglected in both static and dynamical calculations. We define fusion in TDHF as a reaction where a colliding nuclei keeps a compact shape for a sufficiently long time $(\sim 2000 \mathrm{fm} / c)$. More details can be found in Ref. [12].

\section{Result}

\subsection{Nucleus-nucleus potential}

First, we show in Fig. 1 nucleus-nucleus potentials obtained from our method for ${ }^{96} \mathrm{Zr}+{ }^{124} \mathrm{Sn}$, whose charge product is 2,000. The solid and dot-dashed lines are the potentials extracted at $E_{\mathrm{cm}}=250,228 \mathrm{MeV}$, respectively. The filled circles are so-called frozen density potential where the interaction energy is calculated from the energy density functionals with keeping the target and projectile densities frozen to their respective ground-state densities, while all the dynamical effect during the collision and the Pauli effect from the overlap of the projectile and target densities are neglected. One can clearly see that the potentials extracted from our method do not show a barrier structure at smaller distances, and rather show monotonic increase as two nuclei approach to each other. This observation is rather general [12]. We do not observe such a dynamical increase in the potentials extracted in light and medium-mass systems [9] and in the frozen density potentials. We think that this property is related to the consideration that in heavy systems the conditional saddle point appears inside the touching configuration of two nuclei because of large Coulomb energy in the extra-push model [4-6]. Furthermore, we do not see energy dependence of the extracted potential. This is also different from the potential extracted in light and medium-mass systems that show strong energy dependence [9]. Other important effect that increases the nucleus-nucleus potential comes 


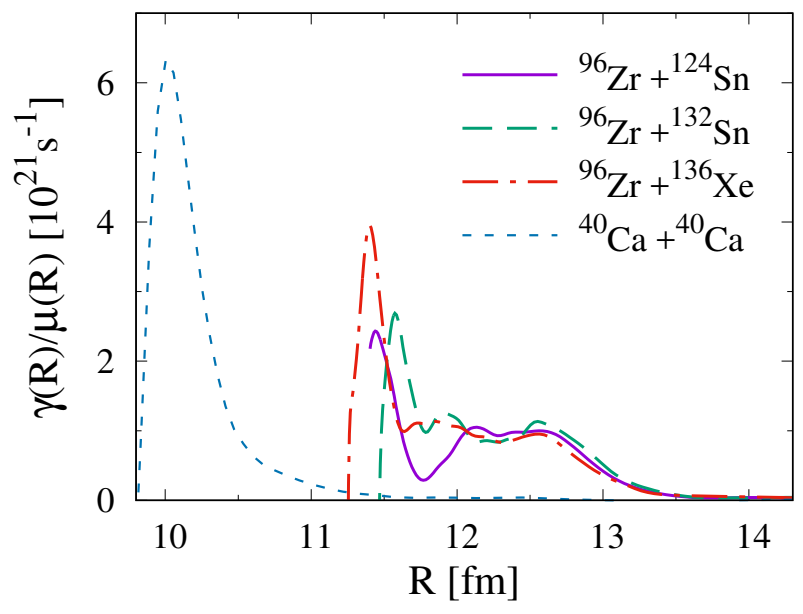

Figure 2. Reduced friction coefficient $\gamma / \mu$ as a function of $R$ for different systems.

from the Pauli effect at overlap of colliding nuclei. As stated above, the Pauli effect is neglected in the frozen density potential, while this is included in TDHF dynamics.

\subsection{Friction coefficient}

As is shown before, our method simultaneously extracts potentials and friction coefficients. Here, we discuss the property of friction coefficients extracted for heavy systems. Figure 2 shows the extracted friction coefficients scaled by the corresponding reduced mass (called reduced friction coefficient) as a function of $R$ for three heavy systems as well as that for medium-mass systems ${ }^{40} \mathrm{Ca}+{ }^{40} \mathrm{Ca}$ as a comparison. Though the extracted potentials in heavy systems are very different from that in light and mediummass systems, the extracted frictions in heavy systems show a similar behavior to that in ${ }^{40} \mathrm{Ca}+{ }^{40} \mathrm{Ca}$, that is, the strength of the friction coefficients in heavy systems is of the same order of magnitude as that in ${ }^{40} \mathrm{Ca}+{ }^{40} \mathrm{Ca}$, and the friction coefficients become larger as $R$ decreases and has a peak around $R_{\min }$.

\subsection{Collective mass}

Our method gives the reduced mass of two-body kinematics during fusion reactions. Figure 3 shows the reduced mass divided by its initial mass $\mu_{0}$ as a function of relative distance scaled by the barrier radius of the frozen density potential denoted by $R_{\mathrm{B}}^{\mathrm{FD}}$ for three heavy systems. At larger $R$, the reduced mass is close to its initial mass, and as $R$ decreases, the reduced mass starts to oscillate and then increase at the distance close to $R_{\min }$. For the ${ }^{70} \mathrm{Zn}+{ }^{208} \mathrm{~Pb}$, sudden decrease is seen after the increase. Thus the reduced mass obtained from our method shows a dynamical property and a relative distance dependence. Recently, the property of the inertial mass for the collective path of nucleus-nucleus collisions has been extensively discussed by the adiabatic self-consistent coordinate method $[14,15]$. In that study, the inertial mass is close to its reduced mass at large $R$, then increases as $R$

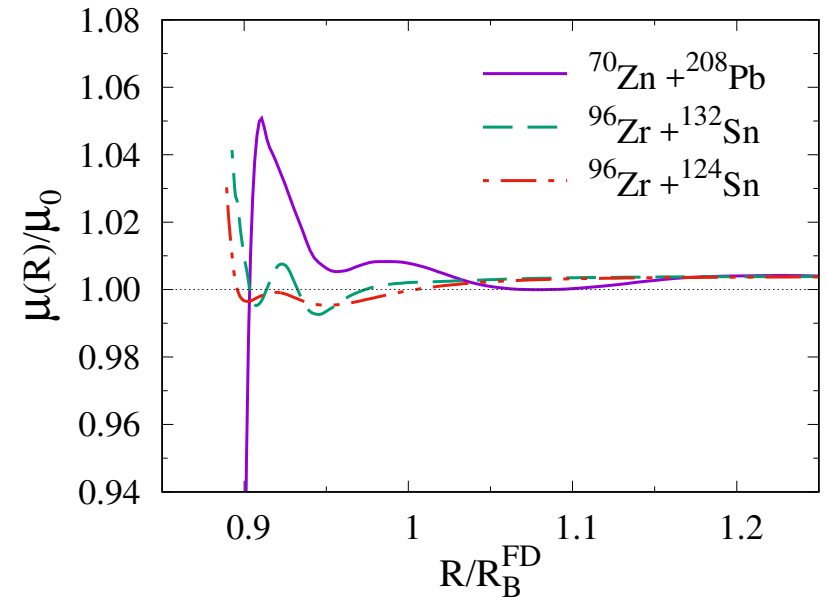

Figure 3. Reduced mass divided by its initial reduced mass as a function of $R$ scaled by its barrier radius for three heavy systems.

decreases, which is similar to the behavior of our reduced mass, though the method of deriving the inertial mass is different from our method.

\subsection{Extra-push energy by TDHF}

TDHF simulations of fusion reactions in heavy systems show fusion hindrance $[12,16,17]$. As a consequence, the extra-push energy $E_{\text {extra }}$ by TDHF can be defined as $E_{\text {extra }}=E_{\text {thres }}-V_{\mathrm{FD}}$, where $E_{\text {thres }}$ is the fusion threshold energy, while $V_{\mathrm{FD}}$ is the barrier height of the frozen density potential. The top panel of Fig. 4 shows the comparison between $E_{\text {extra }}$ by TDHF and the experimental one [18] for the ${ }^{90,92,94,96} \mathrm{Zr}+{ }^{124} \mathrm{Sn}$ systems. Our TDHF simulations underestimate the experimental extra-push energies. The discrepancy becomes larger as the neutron number in $\mathrm{Zr}$ becomes larger. The discrepancy can be attributed by lack of dissipation beyond the mean-field level. (Onebody dissipation is treated self-consistently in our TDHF simulations.) Other reason of this discrepancy might be due to neglecting pairing correlations, which K. Sekizawa pointed out in his talk during the conference.

\subsection{Origin of fusion hindrance}

We have calculated nucleus-nucleus potentials and friction coefficients as well as extra-push energies in heavy systems. Next, we will consider the origin of fusion hindrance. First, we focus on the fusion trajectory at the fusion threshold energy. We will use in the following the nucleus-nucleus potential and friction coefficient extracted at the fusion threshold energy. Then, we define the increase in potential energy $\Delta V$ as the energy between the extracted potential at $R=R_{\min }$ and the barrier $V_{\mathrm{FD}}$ of the frozen density potential, which is $\Delta V=V\left(R_{\min }\right)-V_{\mathrm{FD}}$, and accumulated dissipated energy $E_{\mathrm{diss}}$ at $R=R_{\min }$ as

$$
E_{\mathrm{diss}}\left[R_{\min }(t)\right]=\int_{t_{0}}^{t} d t^{\prime} \gamma\left[R\left(t^{\prime}\right)\right] \dot{R}\left(t^{\prime}\right)^{2},
$$




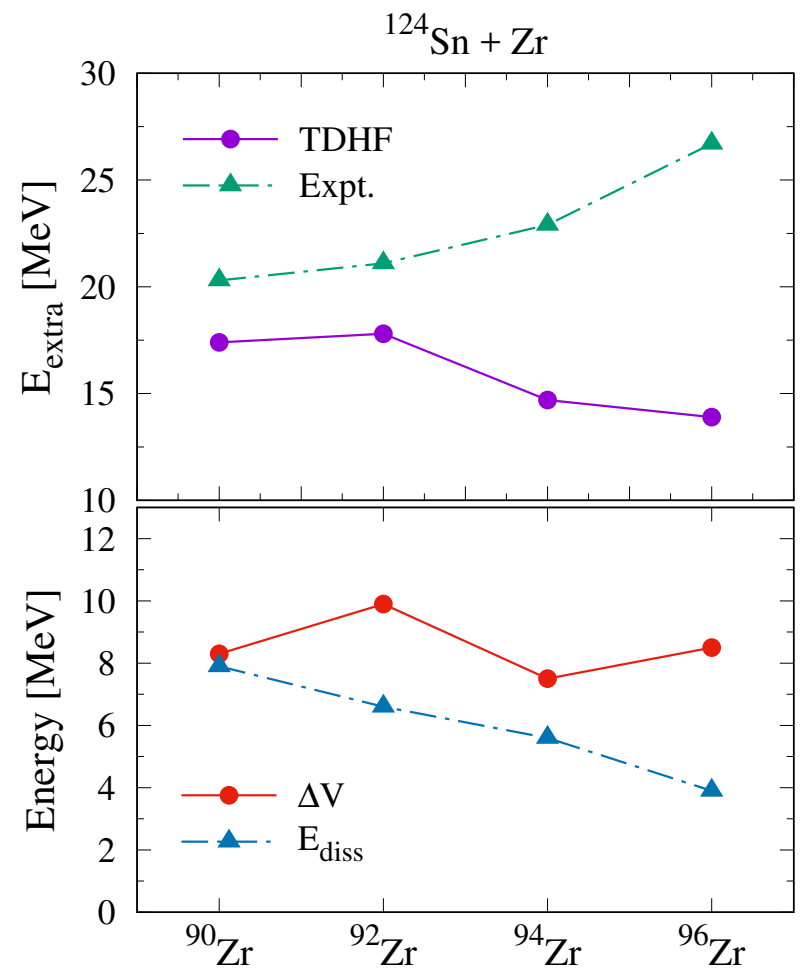

Figure 4. (Top) Comparison between TDHF and experimental [18] extra-push energies. (Bottom) Comparison between the increase in potential energy $\Delta V$ and the accumulated dissipated energy for the ${ }^{90,92,94,96} \mathrm{Zr}+{ }^{124} \mathrm{Sn}$ systems.

where the integration is performed from an initial time $t_{0}$ to the time $t$ at which our extraction procedure stops. From the energy conservation and the fact that the kinetic energy at $R_{\min }$ is very small from our observation, the extra-push energy is simply represented by the sum of the increase in potential energy $\Delta V$ and accumulated dissipated energy $E_{\text {diss. }}$. The bottom panel of Fig. 4 shows the comparison between the increase in potential energy $\Delta V$ and accumulated dissipated energy $E_{\text {diss }}$ for the ${ }^{90,92,94,96} \mathrm{Zr}+{ }^{124} \mathrm{Sn}$ systems. $\Delta V$ is larger than $E_{\text {diss. }}$. We found that $\Delta V>E_{\text {diss }}$ holds for other heavy systems [12]. We conclude that the dynamical increase in potential energy induced from the large Coulomb energy and the Pauli effect at short relative distances is the main contribution to the presence of the extra-push energy and the origin of the fusion hindrance from the present analysis.

\section{Summary}

We have discussed the rather historical fusion hindrance in heavy systems. We performed the analysis of the fusion hindrance by using TDHF dynamics. We extracted nucleus-nucleus potential and energy dissipation from our method combining TDHF dynamics and a macroscopic equation of motion. We showed that potentials in heavy systems show monotonic increase as the relative distance of two nuclei approaches. This is a unique feature seen in heavy systems in our analysis. Then, we analyzed the extra-push energy by using the properties of potentials and energy dissipation extracted from our method. Dynamical increase in potential has more significant contribution to the fusion hindrance.

This work was funded by ImPACT Program of Council for Science, Technology and Innovation (Cabinet Office, Government of Japan).

\section{References}

[1] H. Gäggeler, T. Sikkeland, G. Wirth, W. Brüchle, W. Bögl, G. Franz, G. Herrmann, J. V. Kratz, M. Schädel, K. Sümmerer et al., Z. Phys. A 316, 291 (1984)

[2] C.-C. Sahm, H.-G. Clerc, K.-H. Schmidt, W. Reisdorf, P. Armbruster, F. P. Heberger, J. G. Keller, G. Münzenberg, D. Vermeulen, Z. Phys. A 319, 113 (1984)

[3] C.-C. Sahm, H.-G. Clerc, K.-H. Schmidt, W. Reisdorf, P. Armbruster, F. P. Heberger, J. G. Keller, G. Münzenberg, D. Vermeulen, Nucl. Phys. A 441, 316 (1985)

[4] W. J. Swiatecki, Prog. Part. Nucl. Phys. 4, 383 (1980)

[5] W. J. Swiatecki, Phys. Scr. 24, 113 (1981)

[6] W. J. Swiatecki, Nucl. Phys. A 376, 275 (1982)

[7] Y. Aritomo, M. Ohta, Nucl. Phys. A 744, 3 (2004)

[8] V. Zagrebaev, W. Greiner, J. Phys. G 31, 825 (2005)

[9] K. Washiyama, D. Lacroix, Phys. Rev. C 78, 024610 (2008)

[10] K. Washiyama, D. Lacroix, S. Ayik, Phys. Rev. C 79, 024609 (2009)

[11] P. Bonche, S. Koonin, J.W. Negele, Phys. Rev. C 13, 1226 (1976)

[12] K. Washiyama, Phys. Rev. C 91, 064607 (2015)

[13] K.-H. Kim, T. Otsuka, P. Bonche, J. Phys. G 23, 1267 (1997)

[14] K. Wen, T. Nakatsukasa, Phys. Rev. C 94, 054618 (2016)

[15] K. Wen, T. Nakatsukasa, arXiv:1703.04319 (2017)

[16] L. Guo, T. Nakatsukasa, EPJ Web Conf. 38, 09003 (2012)

[17] C. Simenel, Eur. Phys. J. A 48, 152 (2012)

[18] K.-H. Schmidt, W. Morawek, Rep. Prog. Phys. 54, 949 (1991) 